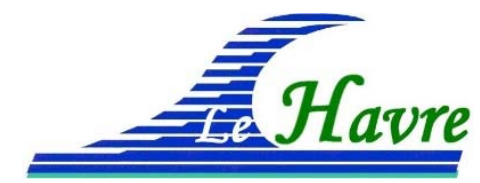

XVI ${ }^{\text {èmes }}$ Journées Nationales Génie Côtier - Génie Civil

Le Havre, 2020

DOI:10.5150/jngcgc.2020.061 C Editions Paralia CFL

disponible en ligne - http://www.paralia.fr - available online

\title{
Devenir sur 10 ans du rechargement massif du Golfe d'Aigues-Mortes
}

\section{Nicolas ALEMAN ${ }^{1}$, Olivier RAYNAL ${ }^{1}$, Raphaël CERTAIN ${ }^{1}$, Nicolas ROBIN ${ }^{1}$, Laurent MONTEL ${ }^{2}$}

\author{
1. CEFREM. UMR5110 Université de Perpignan Via Domitia - CNRS, \\ 58 avenue Paul Alduy, 66860 Perpignan Cedex, France. \\ nicolas.aleman@univ-perp.fr
}

2. DREAL Occitanie. Division Risques Naturels et Littoral, 220 allée Henri II de Montmorency

\section{Résumé :}

Afin de lutter contre l'érosion croissante des plages sableuses du Golfe d'Aigues-Mortes (Occitanie), un rechargement massif en sable a été réalisé pendant l'hiver 2007-2008. Un peu plus d'un million de mètres cube de sables prélevés sur une flèche sous-marine (pointe de l'Espiguette) ont été répartis sur quatre plages soumises à des phénomènes de recul du trait de côte (Palavas, Carnon, Petit Travers et Boucanet). Cet article fait le point sur l'évolution de ce rechargement sur une période de dix ans après l'opération grâce à un suivi topo-bathymétrique annuel. Il met en avant la redistribution spatio-temporelle des sédiments et les relations existantes entre les trois compartiments que sont la plage émergée, la zone de battement du trait de côte et l'avant-côte. Une certaine stabilité semble atteinte seulement quatre ans après le rechargement et le prisme littoral n'a perdu qu'environ $30 \%$ du volume de sable initialement rechargé, mais avec de forte disparité entre les sites. Cette étude devrait permettre aux gestionnaires du littoral de mieux appréhender le devenir d'un rechargement au cours du temps et donc de mieux adapter les volumes à prendre en compte et les compartiments préférentiels à recharger.

\section{Mots-clés :}

Erosion du littoral, Rechargement, Golfe d'Aigues-Mortes, Dynamique littorale, Dragage, Plage émergée, Trait de côte, Avant-côte.

\section{Introduction}

Au début des années 60, la Mission Interministérielle pour l'Aménagement Touristique du Languedoc-Roussillon (mission "Racine", du nom de son coordinateur) prévoit les grandes infrastructures devant capter le tourisme dans la région. Les stations balnéaires de la Grande-Motte et de Carnon sont construites entre 1967 et 1968 et la grande marina de Port Camargue dès 1969. L'érosion côtière qui n'était jusque-là pas un problème commence alors à menacer les infrastructures. De nombreux ouvrages de défense contre la mer (épis et brise-lames) seront installés pour tenter d'endiguer le phénomène. 


\section{Thème 6 - Gestion durable des zones littorales et estuariennes}

Au début des années 2000, les communes du littoral prennent alors conscience de la nécessité de traiter l'érosion dans l'ensemble de la cellule hydrosédimentaire et non pas à l'échelle des zonages administratifs. La création d'un SIVOM permet de diligenter une étude complète qui aboutira à l'élaboration d'un programme de rechargement en sable des plages du Golfe d'Aigues-Mortes. Ce rechargement s'est déroulé de septembre 2007 à avril 2008. A l'aide d'une drague aspiratrice en marche, $1080000 \mathrm{~m}^{3}$ de sédiment ont été prélevés sur la flèche sous-marine de la pointe de l'Espiguette pour être déposés sur un linéaire côtier de $10 \mathrm{~km}$ réparti en quatre secteurs (VANROYE, 2008), voir figure 1 :

- Plage de Palavas à Carnon : $353666 \mathrm{~m}^{3}$ sur $2950 \mathrm{~m}$;

- Plage de Carnon Est : $182546 \mathrm{~m}^{3}$ sur $1750 \mathrm{~m}$;

- Plage du Petit Travers : $373584 \mathrm{~m}^{3}$ sur $3350 \mathrm{~m}$;

- Plage du Boucanet au Grau du Roi : $170210 \mathrm{~m}^{3}$ sur $1985 \mathrm{~m}$.

L'objet de la présente étude est d'évaluer l'évolution du budget sédimentaire des portions de littoral rechargées au cours des dix dernières années (2008-2018) pour évaluer l'efficacité du rechargement sur le long terme. Ces travaux sont issus de l'axe 3 du contrat d'étude PAUL (ALEMAN et al., 2009) dont nous présentons ici les principaux résultats.

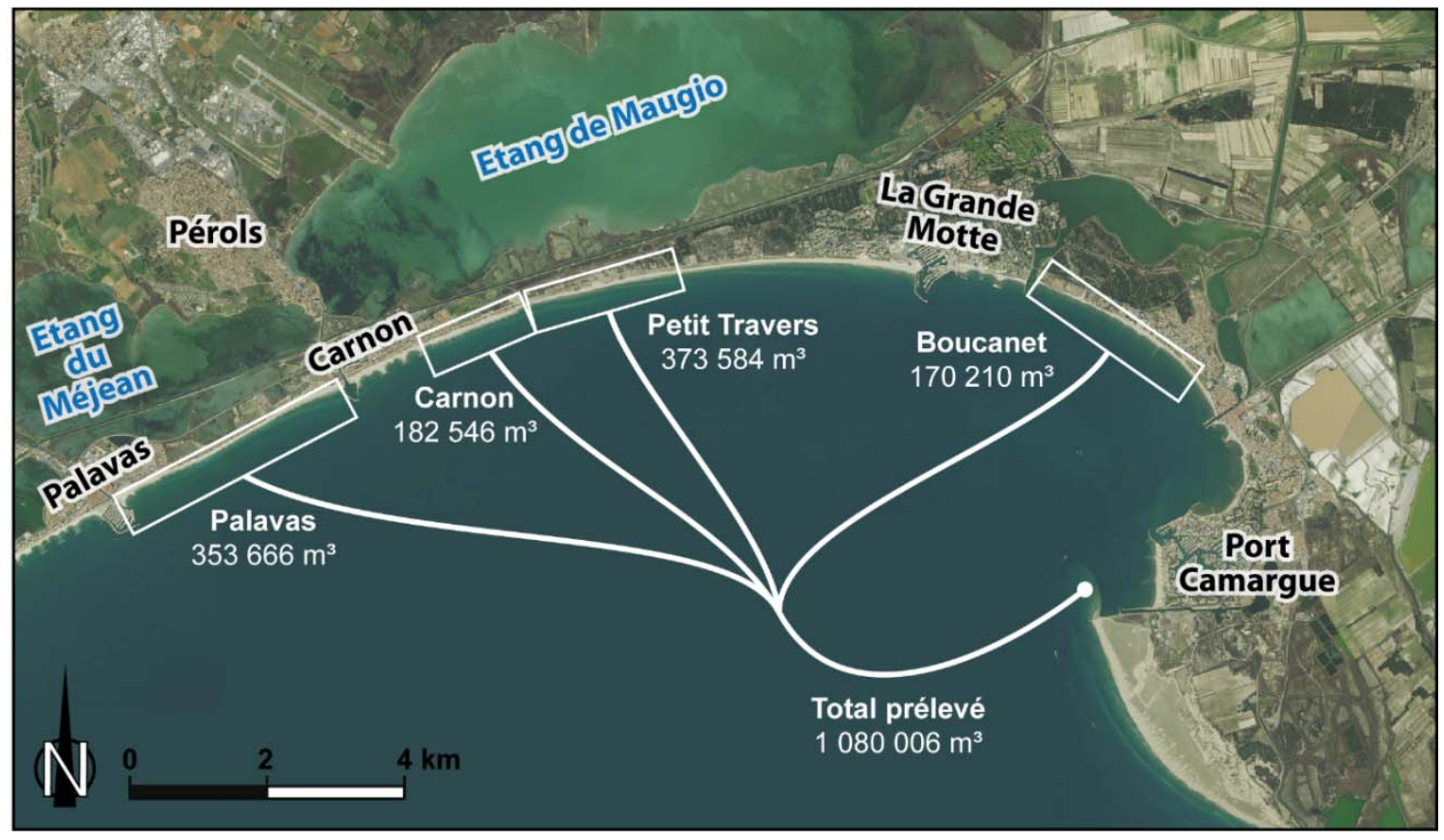

Figure 1. Zones de prélèvement et de rechargement du Golfe d'Aigues-Mortes en 2008.

\section{Méthodologie}

Un suivi du rechargement a été organisé par la DREAL Occitanie avec des campagnes annuelles de levés topo-bathymétriques (réalisées par EID Méditerranée et Semantic TS). L'utilisation d'un DGPS RTK a permis d'atteindre une précision de $\pm 0,2 \mathrm{~m}$ en fonction des conditions et de l'organisation des levés. Les données enregistrées ont permis la 


\section{XVIèmes Journées Nationales Génie Côtier - Génie Civil \\ Le Havre 2020}

réalisation de modèles numériques de terrain sous le logiciel ArcGIS@10.4 par la méthode des voisins naturels. Un modèle numérique de terrain MNT différentiel d'altitude a été construit pour chaque période. La marge d'erreur des MNT différentiels est donnée par l'équation : $\delta_{\text {dif }}=\sqrt{\left(\delta_{M N T 1}\right)^{2}+\left(\delta_{M N T 2}\right)^{2}}$ avec $\delta_{\text {dif }}$ la marge d'erreur du MNT différentiel et, $\delta_{M N T 1}$ et $\delta_{M N T 2}$ les marges d'erreur individuelles des deux MNT. La marge d'erreur intrinsèque des MNT étant de $\pm 0,2 \mathrm{~m}$, la marge d'erreur du MNT différentiel atteint $\pm 0,28 \mathrm{~m}$. Cette valeur sera utilisée par la suite pour calculer la marge d'erreur des budgets sédimentaires du littoral.

Les MNT différentiels ont été découpés selon trois secteurs morphologiques (figure 2) : la plage, le zone de battement du trait de côte et l'avant-côte : la zone "plage" est définie entre le pied de dune ou la limite entre la plage et la zone artificialisée jusqu'à la position du trait de côte la plus reculée à terre entre les deux dates comparées; la zone "trait de côte" est définie entre la position du trait de côte la plus reculée à terre et la position la plus avancée en mer entre les deux dates comparées; la zone "avant-côte" est définie depuis la limite la plus vers le large du trait de côte jusqu'à la limite du levé au large. Pour chaque zone, les structures anthropiques présentes sur la plage lors des levés ont été masquées et une zone tampon de quelques mètres a été prise autour des ouvrages pour éviter les effets de bord.

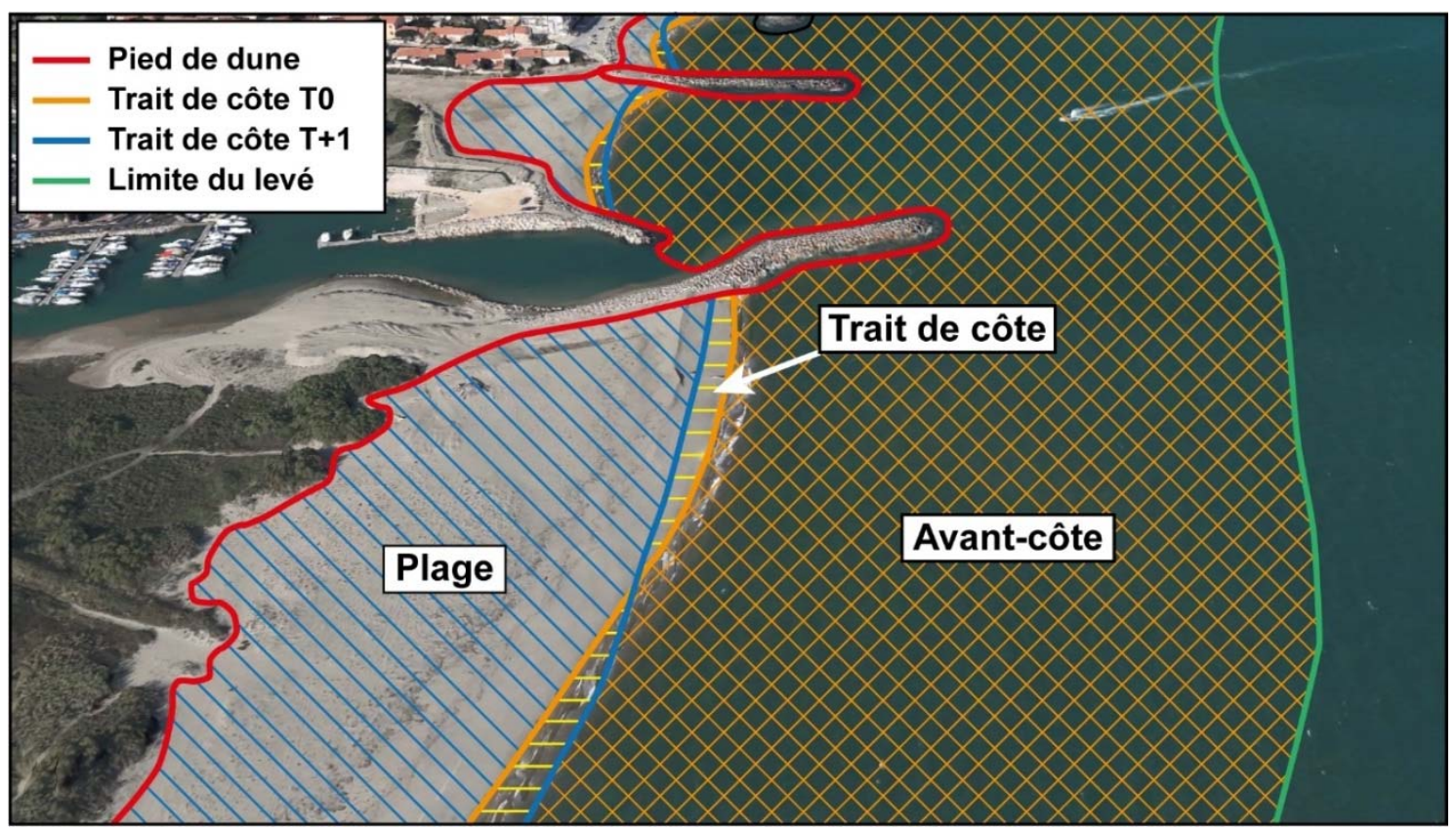

Figure 2. Schéma des différentes zones (plage, trait de côte et avant-côte) et des limites transversales (pied de dune, trait de côte et limite du levé au large utilisées pour le calcul des budgets sédimentaires. 


\section{Thème 6 - Gestion durable des zones littorales et estuariennes}

\section{Résultats}

\subsection{Enregistrement des travaux de dragage et de rechargement}

En 2008, après les travaux de rechargement, la flèche sous-marine de l'Espiguette perd un volume de sable correspondant sensiblement au volume prélevé pour le rechargement $\left(-883260 \pm 96715 \mathrm{~m}^{3}\right)$ (figure 3). La différence avec le volume dragué annoncé par le prestataire $\left(1080006 \mathrm{~m}^{3}\right)$ peut s'expliquer par le comblement rapide de la zone de prélèvement qui a certainement déjà commencé (e.g. gain de $680810 \pm 88552 \mathrm{~m}^{3}$ l'année suivant le prélèvement) et par la précision intrinsèque de la donnée de terrain. Sur les quatre plages rechargées, le prisme sableux enregistre un gain total d'un peu plus de $900000 \mathrm{~m}^{3}$ de sédiment $\left(270000 \pm 6000 \mathrm{~m}^{3}\right.$ à Palavas, $166000 \pm 2000 \mathrm{~m}^{3}$ à Carnon, $367000 \pm 6000 \mathrm{~m}^{3}$ au Travers et $108000 \pm 11000 \mathrm{~m}^{3}$ au Boucanet), soit environ 169000 $\mathrm{m}^{3}$ de moins que le volume annoncé par le prestataire (différence de $84000 \mathrm{~m}^{3}$ à Palavas, $66000 \mathrm{~m}^{3}$ à Carnon, $6000 \mathrm{~m}^{3}$ au Travers et $63000 \mathrm{~m}^{3}$ au Boucanet). Une partie des sables rechargés a certainement déjà été redistribué de part et d'autre des cellules suivies entre les deux levés.

\subsection{Evolution du littoral post-travaux}

Sur les quatre secteurs, la plage émergée (entre le trait de côte et le pied de dune) perd la totalité du sédiment rechargé en seulement deux ans (2009-2010). A Palavas, les pertes se poursuivent encore un an avant de se stabiliser au tour de $-40000 \mathrm{~m}^{3}$ par rapport à 2008 avant les travaux (figure 3). A Carnon, la plage émergée se stabilise avec le même volume qu'avant le rechargement. Au Travers et au Boucanet, la plage regagne progressivement des sédiments jusqu'à atteindre à nouveau en 2016 le volume rechargé d'origine.

La zone de battement du trait de côte de Palavas, Carnon et Boucanet perd environ la moitié des sédiments rechargés pendant les deux années suivant le rechargement avant de se stabiliser. La plage du Travers perd seulement un quart des sédiments avant de se stabiliser. En 2018, le gain sédimentaire restant dans la zone du trait de côte après le rechargement de 2008 est d'environ $31000 \pm 360 \mathrm{~m}^{3}$ à Palavas, $46000 \pm 250 \mathrm{~m}^{3}$ à Carnon, $126000 \pm 760 \mathrm{~m}^{3}$ au Travers et $25000 \pm 210 \mathrm{~m}^{3}$ au Boucanet.

L'évolution du budget sédimentaire de l'avant-côte est plus hétérogène. L'avant-côte continue à gagner du sédiment un an après le rechargement, certainement en raison de la redistribution des sédiments perdus par la plage et le trait de côte (attention, le très fort gain puis la perte observée à Carnon entre 2009 et 2010 semble plutôt provenir d'un problème de calage de la donnée comme le laisse suggérer l'étalement des sédiments sur les différentiels). Par la suite, l'avant-côte perd du sédiment pendant un à trois ans avant de se stabiliser ou de regagner du sable, notamment entre 2015 et 2016, où l'ensemble des plages sont en accrétion (figure 3) suite aux pertes liées à la forte tempête du $24 / 12 / 2013$. 


\section{XVI èmes Journées Nationales Génie Côtier - Génie Civil \\ Le Havre 2020}
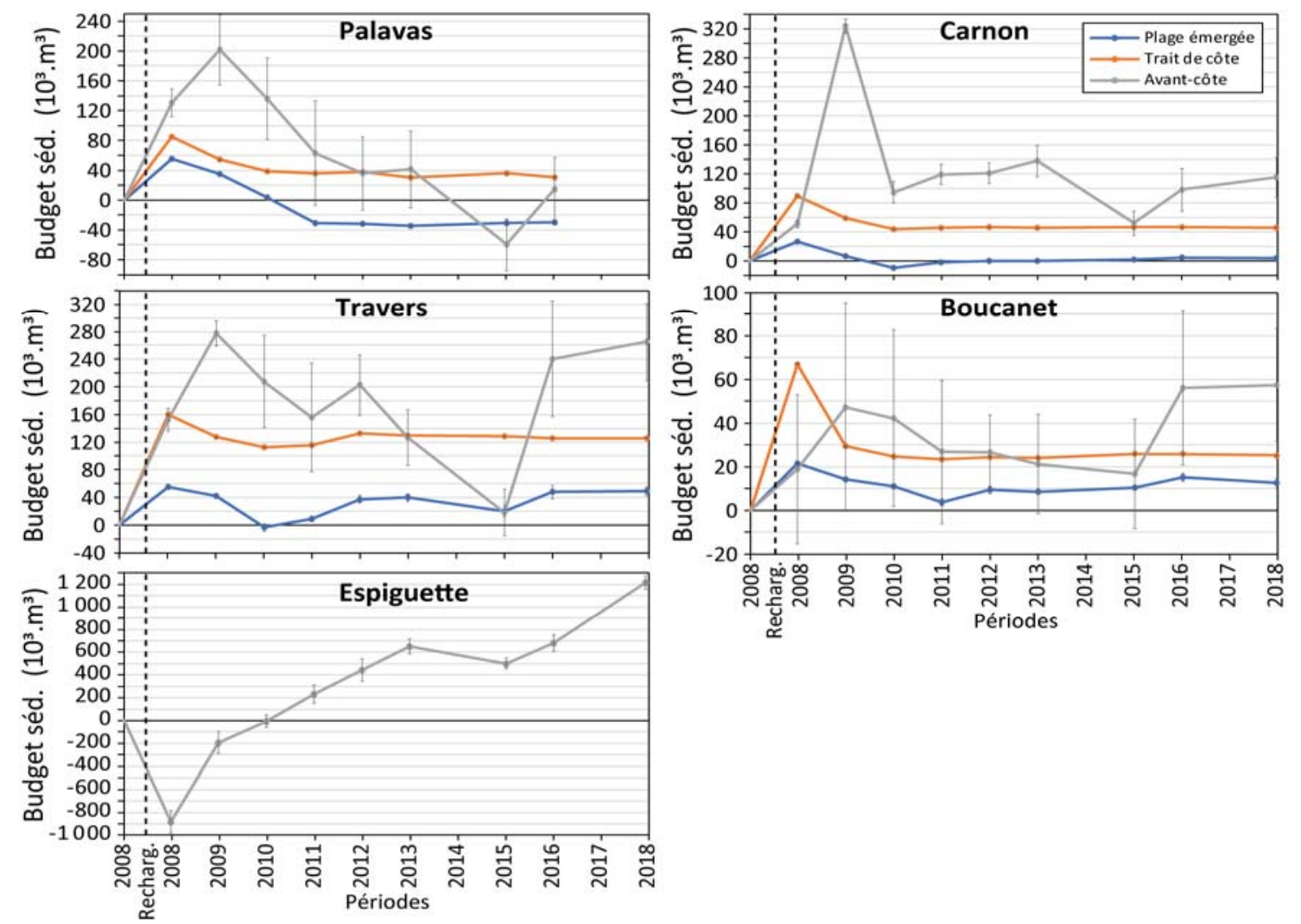

Figure 3. Budget sédimentaire cumulé entre 2008 et 2018 de la plage émergée, de la zone de battement du trait de côte et de l'avant-côte pour les quatre plages rechargées (Palavas, Carnon, Travers et Boucanet) et de la source (Espiguette).

\subsection{Répartition spatiale des sédiments}

Les MNT différentiels montrent qu'entre 2008 et 2009, entre un tiers et la moitié du volume de sédiments rechargé est redistribué vers l'avant-côte et le système de barres sédimentaires. Entre 2009 et 2010, même si une partie des sédiments doit être transportée longitudinalement via la dérive littorale, l'érosion généralisée du prisme littoral sur toutes les cellules voisines les unes des autres souligne un transport vers le large au-delà de la zone de levé. Ces pertes se poursuivent en 2011 à Palavas et au Boucanet. A partir de 2010 , on observe une stabilisation du système qui retrouve un fonctionnement équilibré. Seule l'avant-côte présente des variations importantes de son budget sédimentaire, les mouvements se concentrant principalement dans le système de barres sédimentaires d'avant-côte. Entre 2013 et 2015 notamment, l'avant-côte subit une forte érosion (entre $780 \pm 9400 \mathrm{~m}^{3}$ et $-129000 \pm 12600 \mathrm{~m}^{3}$ ) compensée par un retour de sédiments entre 2015 et 2016 (entre $39500 \pm 45700 \mathrm{~m}^{3}$ et $222600 \pm 134600 \mathrm{~m}^{3}$ ). Cette forte érosion de l'avantcôte traduit un transfert de sédiments vers le large au-delà de la zone de suivi, avant une remontée des sables vers le système de barres ensuite. La perte de sédiments peut être liée à la tempête du 24/12/2013 dont les hauteurs significatives de la houle ont atteint 4,6 m, 


\section{Thème 6 - Gestion durable des zones littorales et estuariennes}

les plus importantes enregistrées depuis la mise en service de l'houlographe de l'Espiguette en 2008, le retour de sable se faisant ensuite en raison de l'asymétrie de la houle lors de conditions de forçages plus habituelles.

\section{Interprétation}

\subsection{Evolution globale du rechargement}

Ces résultats montrent que le prisme sableux retrouve un fonctionnement naturel, composé d'échanges avec le glacis sans pertes majeures pour la partie supérieure (plage émergée et trait de côte), seulement deux à trois ans après le rechargement (tableau 1). Les sédiments de la plage et du trait de côte sont par contre en partie exportés vers l'avantcôte dès la première année suivant le rechargement, traduisant une réaction rapide de réajustement de la plage émergée suite au rechargement. Deux ans après le rechargement, l'ensemble du prisme sableux passe en érosion par rapport à l'année précédente, une partie des sédiments est transportée longitudinalement par rapport à la côte par la dérive littorale mais les principales pertes semblent s'effectuer vers le large car elles ne sont plus détectables dans les secteurs étudiés adjacents les uns des autres. Trois ans après le rechargement, des pertes de sédiment subsistent mais le littoral retrouve une certaine stabilité. Quatre ans après, la plage et le trait de côte se stabilisent définitivement et l'avant-côte retrouve un fonctionnement naturel composé d'une alternance de phases d'érosion et d'accrétion liées aux conditions climatiques (tableau 1). On note que certaines conditions hydrodynamiques sont capables de faire remonter vers l'avant-côte le sédiment qui est sorti de la zone d'étude comme entre 2015 et 2016.

Tableau 1. Evolution synthétique du rechargement en quatre ans.

\begin{tabular}{|c|c|c|c|c|}
\hline \multirow{2}{*}{ Temps } & \multicolumn{3}{|c|}{ Comportement } & \multirow{2}{*}{ Observation } \\
\hline & Plage & Trait de côte & Avant-côte & \\
\hline TO & Gains & Gains & Gains & Rechargement \\
\hline$T+1$ ans & Pertes & Pertes & Gains & Transfert vers l'avant-côte \\
\hline$T+2$ ans & Pertes & Pertes & Pertes & $\begin{array}{l}\text { Transfert vers le large (et } \\
\text { longitudinal) }\end{array}$ \\
\hline$T+3$ ans & Pertes/Stables & Pertes/Stables & Pertes/Stables & $\begin{array}{l}\text { Transfert vers le large (et } \\
\text { longitudinal) }\end{array}$ \\
\hline $\begin{array}{l}T+4 \text { ans } \\
e t+\end{array}$ & Stable & Stable & $\begin{array}{l}\text { Variations } \\
\text { naturelles }\end{array}$ & Retour à l'équilibre naturel \\
\hline
\end{tabular}




\section{XVI'mes Journées Nationales Génie Côtier - Génie Civil \\ Le Havre 2020}

\subsection{Durabilité du rechargement}

Dix ans après le rechargement massif du Golfe d'Aigues-Mortes, le prisme sableux a gardé $714500 \pm 41250 \mathrm{~m}^{3}$ de sédiment sur les $911200 \mathrm{~m}^{3}$ enregistrés après le rechargement de 2008. Le prisme littoral n'a perdu que $196700 \mathrm{~m}^{3}$ en dix ans, soit $22 \%$ du volume initialement rechargé (34\% si l'on considère le chiffre donné par le prestataire de $1080006 \mathrm{~m}^{3}$ ). La perte la plus importante a lieu à Palavas qui a perdu $94 \%$ du volume initial en dix ans. Toutefois, cette perte sédimentaire a lieu principalement sur la plage émergée, le trait de côte conserve lui un gain de $30600 \pm 360 \mathrm{~m}^{3}$. La zone du Boucanet ne perd que $11 \%$ du volume total rechargé et le trait de côte a gardé un volume de 25200 $\pm 210 \mathrm{~m}^{3}$. Le secteur de Carnon a gardé la totalité du volume rechargé dix ans après, avec un gain de $45700 \pm 250 \mathrm{~m}^{3}$ pour le trait de côte. Enfin, le littoral du Travers a gagné plus de sédiments que ce qui a été rechargé à l'origine $\left(+72280 \mathrm{~m}^{3}\right)$. Ces chiffres semblent donc indiquer la bonne durabilité du rechargement dans le temps notamment au niveau du volume sédimentaire de la zone de battement du trait de côte. Cette pérennité peut s'expliquer par le caractère semi-fermé du Golfe d'Aigues-Mortes et la présence de nombreux ouvrages de défense qui fixent les sédiments.

\subsection{Evolution de la source}

La flèche sableuse sous-marine de la pointe de l'Espiguette récupère en seulement deux ans la totalité des sédiments dragués pour le rechargement. Par la suite, la flèche sousmarine gagne en moyenne $200000 \mathrm{~m}^{3}$ de sable par an. Ces valeurs sont proches de celle $\mathrm{du}$ transport sédimentaire longitudinal résiduel calculé par modélisation qui atteint $160000 \mathrm{~m}^{3} / \mathrm{an}$ au droit de la digue d'arrêt des sables (KULLING \& SABATIER, 2015). On observe toutefois une perte de $151600 \pm 24250 \mathrm{~m}^{3}$ entre 2013 et 2015 qui correspond à deux dragages d'un total de $600000 \mathrm{~m}^{3}$ environ. Ce prélèvement est rapidement comblé par les apports massifs dès 2015.

\subsection{Relation entre volume sédimentaire et position du trait de côte}

La position du trait de côte constitue un des principaux indicateurs de l'état du prisme sableux utilisés par les gestionnaires, puisqu'il conditionne la largeur et donc la surface de plage disponible. Cette étude centrée sur l'évolution du budget sédimentaire du littoral, c'est-à-dire du volume de sédiment, met en avant la préservation d'une bonne partie du rechargement au cours du temps, notamment dans la zone de battement des traits de côte. Il est important de souligner ici qu'un volume sédimentaire positif ne reflète pas forcément une position du trait de côte plus avancée en mer. En effet, les gains et pertes sédimentaires sont généralement localisés et, par exemple, un secteur caractérisé par une forte accrétion contre un ouvrage et de l'érosion diffuse sur tout le reste de la zone génère un bilan positif mais une rétrogradation du trait de côte sur une grande partie de la zone. De plus, un trait de côte peut rétrograder de plusieurs mètres tout en ayant une différence altimétrique faible, masquant donc l'érosion dans la marge d'erreur. 


\section{Thème 6 - Gestion durable des zones littorales et estuariennes}

Pour exemple, la figure 4 présente la position du trait de côte de la plage du Boucanet au cours du temps par rapport à celle de 2008 avant les travaux de rechargement. Juste après les travaux, le trait de côte montre une forte progradation consécutive au rechargement. Jusqu'en 2015, la position du trait de côte oscille autour d'une position légèrement plus avancée qu'avant les travaux. Enfin, le trait de côte se retrouve dans une position plus dégradée qu'avant les travaux à partir de 2016 avec un recul moyen de $11,8 \mathrm{~m}$ sur la zone. Ce recul part rapport à 2008 avant les travaux est hétérogène spatialement avec quatre secteurs localisés en progradation de 5 à $10 \mathrm{~m}$ et la grande majorité de la zone en érosion avec des reculs pouvant atteindre jusqu'à $20 \mathrm{~m}$. Pourtant, les bilans sédimentaires soulignent un gain résiduel d'environ $25000 \mathrm{~m}^{3}$ pour la zone de battement du trait de côte. En effet, malgré la rétrogradation du trait de côte sur une grande partie de cette portion de littoral, l'érosion en termes de volume est peu visible (une tache d'érosion au nord-ouest de la zone sur le MNT différentiel) car comprise en majorité dans la marge d'erreur. Au final, ce sont les quelques pixels en forte accrétion qui génèrent un bilan sédimentaire résiduel positif. Les deux techniques d'analyse sont donc complémentaires.

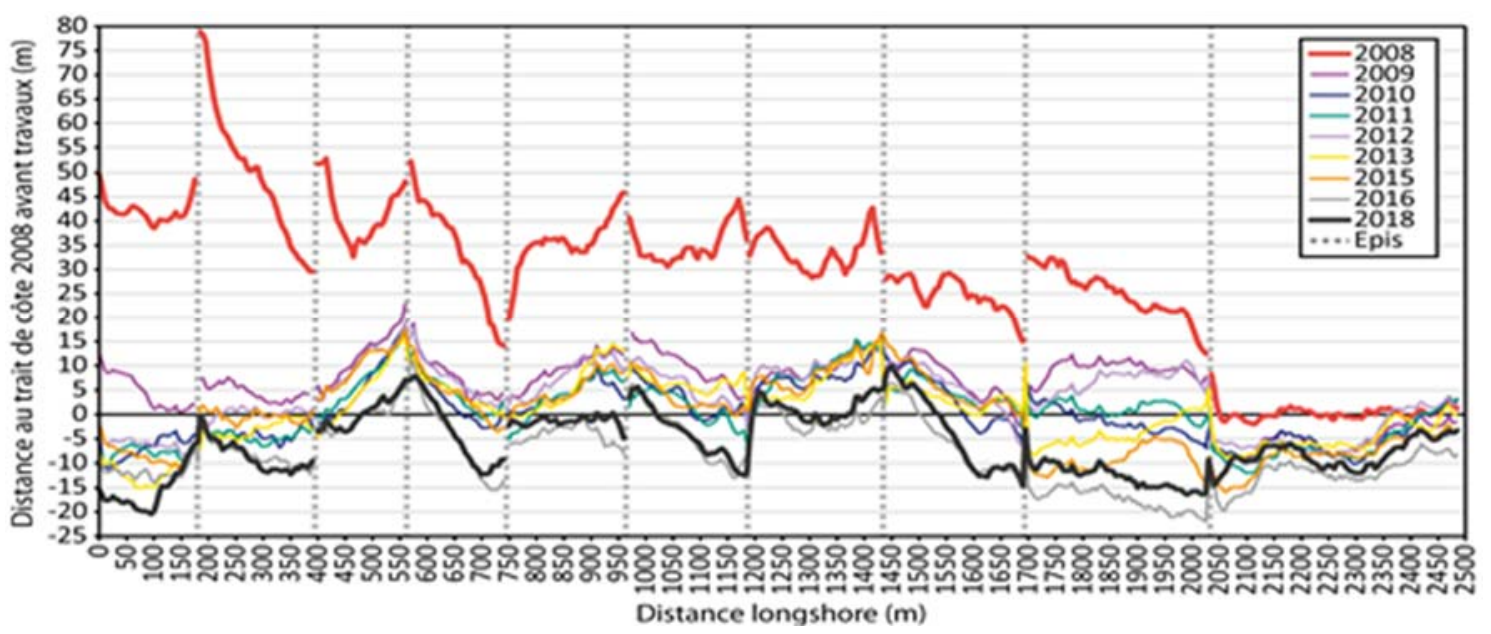

Figure 4. Evolution de la position du trait de côte de la cellule du Boucanet entre 2008 après travaux et 2018 en fonction de la position en 2008 avant travaux.

\section{Conclusion et perspectives}

Cette étude a permis de faire le point sur le devenir, en termes de volume sédimentaire, du rechargement massif du Golfe d'Aigues-Mortes sur une période de 10 ans. Elle montre que les sédiments sont redistribués rapidement vers l'avant-côte mais qu'un équilibre et une stabilité sont atteints quatre ans après le rechargement. Seul 22 à 34\% des sédiments rechargés ont quitté la zone d'étude, principalement vers le large. La flèche sous-marine de l'Espiguette utilisée comme source de sédiments récupère très rapidement (2 ans) l'ensemble du volume dragué et poursuit son accrétion. Il s'agit donc d'un site de choix comme source de sédiments pour de futurs rechargements. 


\section{XVIèmes Journées Nationales Génie Côtier - Génie Civil \\ Le Havre 2020}

Cependant, la persistance des sédiments sur la zone rechargée ne se traduit pas forcément par une avancée du trait de côte par rapport à sa position avant les travaux de rechargement. Il est important de poursuivre ce travail par une étude fine des variations du trait de côte sur la même période. Une confrontation avec la présente étude permettra certainement aux gestionnaires d'apprécier l'efficacité dans le temps du rechargement (en termes de gain de surface de plage) et de déterminer encore plus précisément les zones et la fréquence des réinterventions pour maintenir une position du trait de côte déterminée.

\section{Remerciements}

Nous remercions la DREAL Occitanie pour son soutien financier pour la réalisation de cette étude.

\section{Références bibliographiques}

ALEMAN N., RAYNAL O., CERTAIN R. (2019). Evolution du rechargement massif des plages du Golfe d'Aigues-Mortes (2008-2018). Rapport axe 3 - PAUL, 66 p.

KULliNG B., SABATIER F. (2015). Atlas du Potentiel de transport sédimentaire longitudinal résultant annuel de l'avant-côte du Languedoc-Roussillon. Dreal-LR / Cerege, $18 \mathrm{p}$.

VANROYE C. (2008). La protection du littoral du Golfe d'Aigues-Mortes. X ${ }^{\mathrm{ème}}$ Journées Nationales Génie Côtier - Génie Civil, Sophia Antipolis, pp. 303-312. https://doi.org/10.5150/jngcgc.2008.029-V 
Thème 6 - Gestion durable des zones littorales et estuariennes 